\title{
miR-92a is a critical regulator of the apoptosis pathway in glioblastoma with inverse expression of BCL2L11
}

\author{
HUANJIANG NIU $^{1 *}$, KUN WANG $^{2 *}$, ANLING ZHANG $^{3,4}$, SHUXU YANG $^{1}$, ZHENGFEI SONG $^{2}$, \\ WEI WANG ${ }^{5}$, CONG QIAN $^{1}$, XINWEI LI $^{1}$, YINXIN ZHU ${ }^{1}$ and YIRONG WANG ${ }^{1}$
}

\begin{abstract}
${ }^{1}$ Department of Neurosurgery, Sir Run Run Shaw Hospital, Medical College, Zhejiang University, Hangzhou 310016;
${ }^{2}$ Department of Neurosurgery, Hangzhou Xiasha Hospital, Sir Run Run Shaw Hospital, Medical College,

Zhejiang University, Hangzhou 310016; ${ }^{3}$ Department of Neurosurgery, Tianjin Medical University General Hospital,

Tianjin 300052; ${ }^{4}$ Laboratory of Neuro-oncology, Tianjin Neurological Institute, Tianjin 300052; ${ }^{5}$ Department of Pharmacy,

Hangzhou Xiasha Hospital, Sir Run Run Shaw Hospital, Medical College, Zhejiang University, Hangzhou 310016, P.R. China
\end{abstract}

Received June 5, 2012; Accepted July 13, 2012

DOI: 10.3892/or.2012.1970

\begin{abstract}
Recent studies have revealed that miR-92a is overexpressed in several types of malignancies and provides a protumorigenic effect. Our findings demonstrate that the high expression of miR-92a in human glioma specimens is significantly correlated with low levels of BCL2L11 (Bim) protein and high-grade glioma. Here, we present the first evidence that miR-92a antisense oligonucleotide (AS-miR-92a) provides a tumor suppressive effect via induction of apoptosis in human glioma cells. In addition, we show that Bim is a direct functional target of miR-92a. Introducing Bim cDNA without 3'UTR abrogates miR-92a-induced cell survival. Further investigations will focus on the therapeutic use of AS-miR-92a-mediated antitumor effects in glioma.
\end{abstract}

\section{Introduction}

Malignant glioma is one of the most common and fatal types of brain tumors in humans, which are notoriously difficult to treat and recurrences arise virtually in every case $(1,2)$. Despite new biological insights and advances in therapy, the prognosis of patients with gliomas still remains poor in the last four decades $(3,4)$. Recent studies have developed a novel, molecularly-targeted, multimodal therapeutic approach with the use of antisense 'antagomir' oligonucleotides (5), which may enable development of novel cancer therapies.

Correspondence to: Professor Yirong Wang, Department of Neurosurgery, Sir Run Run Shaw Hospital, Medical College, Zhejiang University, Hangzhou 310016, P.R. China

E-mail: wang.yr@163.com

*Contributed equally

Key words: glioma, miR-92a, Bim, apoptosis
miRNAs are small, evolutionary conserved RNA molecules that control gene expression through binding to the seed sequence at the 3'UTR of target mRNAs, resulting in translational repression or mRNA degradation $(6,7)$. They are involved in diverse biological processes, such as development, differentiation, cell proliferation and apoptosis $(8,9)$. Recent studies have shown that the expression of many miRNAs are altered in various human tumors and some miRNAs may function as oncogenes or tumor suppressor genes (10-13). Recent studies showed that miR-92a, a member of miR-17-92 cluster was frequent deregulated in a variety of cancer types (14-17). So far, there are few reports on miR-92a in glioma.

In our study, we analyzed the expression of miR-92a both in glioma cell lines and samples. We demonstrated that downregulation of miR-92a significantly induced apoptosis. In addition, we demonstrated that Bim was the direct target of miR-92a and played an important role in the miR-92a induced apoptosis. Furthermore, we also showed that miR-92a inversely correlated with Bim expression in glioma tissues. These results identify a critical role for miR-92a in regulation of proliferation and apoptosis in glioma, suggesting that miR-92a could be critical therapeutic target for glioma intervention.

\section{Materials and methods}

Cell culture and culture conditions. The human U251, U87, LN229, U87 and A172 glioblastoma cell lines were purchased from the Institute of Biochemistry and Cell Biology, Chinese Academy of Science. All cells were maintained in a $37^{\circ} \mathrm{C}$, $5 \% \mathrm{CO}_{2}$ incubator in Dulbecco's modified Eagle's medium (DMEM) (Gibco, Carlsbad, CA, USA) supplemented with $10 \%$ fetal bovine serum (Invitrogen, Carlsbad, CA, USA).

Human glioma samples. Human glioma samples were obtained from the Department of Neurosurgery, Sir Run Run Shaw Hospital, Medical College, Zhejiang University after informed consent from adult patients diagnosed with glioblastoma, freshly resected during surgery and immediately frozen in liquid nitrogen for subsequent total RNA extraction. 
RNA extraction and quantitative real-time PCR. Total RNA was isolated from cultured cells, human glioma specimens, or NAT (normal adjacent tissue)s using TRIzol ${ }^{\circledR}$ reagent (Invitrogen) according to the manufacturer's instructions. Quantitative real-time polymerase chain reaction (qRT-PCR) was performed in triplicate in ABI 7500HT fast real-time PCR system (Applied Biosystems, Foster City, CA, USA) and normalized with U6 and glyceraldehyde 3-phosphate dehydrogenase (GAPDH) endogenous control. Total RNA from NATs was used as a control. miR-92a levels were measured with the TaqMan microRNA assay kit, and endogenous mRNA levels of Bim were detected using SYBR-Green PCR Master Mix kit in accordance with the manufacturer's instructions (Applied Biosystems).

Oligonucleotide, Bim expression plasmid synthesis and transfection. miR-92a: 5'-UAUUGCACUUGUCCCGGCCUGU-3', AS-miR-92a: 5'-ACAGGCCGGGACAAGUGCAAUA-3' and scrambled control oligonucleotides were purchased from Shanghai GenePharma Co., Ltd. (Shanghai, China). Bim expression plasmid was preserved in our laboratory. miRNA sequences/Bim expression plasmid were transfected into cultured cells using Lipofectamine 2000 reagent (Invitrogen) following the manufacturer's instructions.

Cell growth assays. The MTT assay was used to determine relative cell growth as follows. U251 and U87 cells were plated at $10^{4}$ cells/well in 96-well plates with 6 replicate wells for each condition, transfected with oligonucleotides, and assayed 48 -h post-transfection. Cell growth assay was performed by MTT (Sigma, St. Louis, MO, USA) as previously described (18). The cell viability was determined at 540-nm absorbance using an enzyme-linked immunosorbent assay plate reader. All data points represent the mean of a minimum of 6-wells.

Apoptosis assay. Forty-eight hours after transfection, apoptosis in cultured cells was evaluated with Annexin V labeling, caspase-3/7 activity and mitochondrial membrane potential. For the Annexin V assay, an Annexin V-FITC labeled Apoptosis Detection kit (Abcam) was used according to the manufacturer's protocol. Caspase-3/7 activity was measured using caspase-Glo-3/7 reagent (Promega, Madison, WI, USA). Mitochondrial membrane potential was determined with cationic dye JC-1 (5,5',6,6'-tetrachloro-1,1',3,3'-tetraethylbenzi-midazolylcarbocyanine-chloride/C25H27C13N4) staining (19). Briefly the cells were harvested and first stained with PI. Following wash twice with PBS, the cells were incubated with $10 \mathrm{mg} / \mathrm{ml} \mathrm{JC}-1$ for $20 \mathrm{~min}$ at room temperature and then analyzed with FACSCalibur to detect green fluorescence at excitation/emission wavelengths of $485 / 530 \mathrm{~nm}$ and red fluorescence at excitation/emission wavelengths of 485/590 nm. TUNEL assay was used to detect the apoptosis in tumor specimens and was performed as previously described (20).

Western blot analysis, miRNA locked nucleic acid(LNA) in situ hybridization, immunohistochemistry and luciferase reporter assay. Western blot analysis, miRNA-LNA in situ hybridization and immunohistochemistry were performed as previously described (21). For reporter assay, the pGL3-WT-Bim-3'UTR-
Luc reporter (WT Bim) was created by the ligation of Bim 3'UTR PCR products into the $X b a \mathrm{I}$ site of the pGL3 control vector (Promega). The pGL3-MUT-Bim-3'UTR-Luc reporter (MUT Bim) was generated from pGL3-WT-Bim-3'UTR-Luc by replacing the binding site of miR-92a with restriction enzyme cutting site CGGATCCG. For the reporter assay, cells were cultured in 96-well plates and transfected with WT Bim/ MUT Bim and AS-miR-92a. Luciferase activity was measured $48 \mathrm{~h}$ after transfection with the Dual-luciferase reporter assay system. The Renilla luciferase activity was utilized as an internal control.

Nude mouse tumor xenograft model and AS-miR-92a treatment. U251 glioma cells were subcutaneously injected to 5-week-old female nude mice (Cancer Institute of The Chinese Academy of Medical Science). When the tumor volume reached $100 \mathrm{~mm}^{3}$, the mice were randomly divided into two groups (10 mice per group) which were treated with 200 pmol scramble oligo or AS-miR-92a in $10 \mu$ l Lipofectamine 2000 through local injection of xenograft tumor in multiple sites. The treatment was performed once every 2 days for 14 days. The tumor volume was measured with a caliper every 2 days, using the formula: volume = length $\mathrm{x}$ width $2 / 2$.

Statistical analysis. All tests were done using SPSS Graduate Pack 11.0 statistical software (SPSS Inc., Chicago, IL, USA). Statistical evaluation for data analysis was determined by one-way ANOVAs, the Student's t-test and the Chi-square test. Differences with $\mathrm{P}<0.05$ were considered statistically significant.

\section{Results}

miR-92a is overexpressed in human glioma. To explore the expression of miR-92a in human glioma samples and cell lines, we performed the TaqMan-based real-time stem-loop RT-PCR analyses. Our data showed that miR-92a was obviously upregulated in glioma cell lines vs. NATs (Fig. 1A). Similar results were observed in the glioma tissues (Fig. 1B). Taken together, our results demonstrated that miR-92a was abnormally overexpressed both in human glioma samples and cell lines.

AS-miR-92a suppresses cell proliferation and induces apoptosis in glioma cell lines. To examine biological significance of miR-92a in glioma, U251 and U87 cells were treated with AS-miR-92a and scramble oligonucleotides (Fig. 1B). First of all, real-time PCR showed that AS-miR-92a reduced miR-92a levels by $81 \%$ in U251 cells and $74 \%$ in U87 cells $(\mathrm{P}<0.05)$ (Fig. 2A). MTT assay showed that proliferation was inhibited in the miR-92a abrogation glioma cells, which was the most apparent on Day 3 (Fig. 2B). We further explored effects of AS-miR-92a on apoptosis. Annexin V labeling revealed that knockdown of miR-92a significantly increased cell apoptosis compared to the cells treated with scramble oligonucleotide and control group (Fig. 2C). Moreover, western blot assay displayed that pro-apoptotic protein Bax and cleaved-caspase-3 expression were significantly upregulated while Bcl-2 expression was downregulated in As-miR-92a group (Fig. 2F). In addition, caspase-3/7 activity 
A

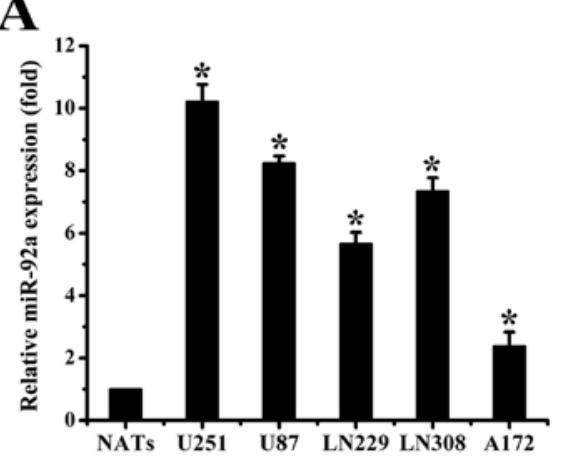

B

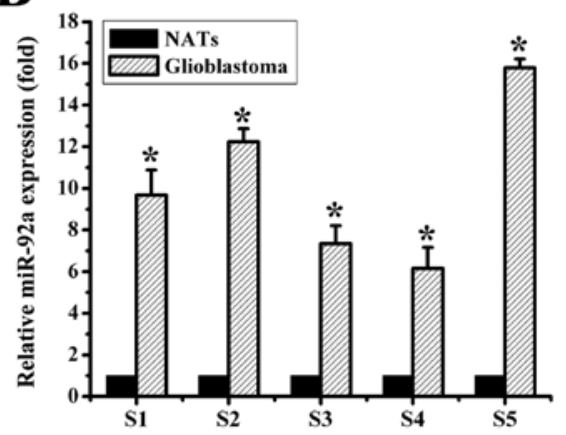

Figure 1. miR-92a shows overexpression in glioma cell lines and samples. (A) miR-92a expression in glioma cells (U251, U87, LN229, U87 and A172) and NATs by real-time PCR. (B) qRT-PCR analysis showed that glioblstoma tissues express much higher levels of miR-92a compared with NATs. Data are mean $( \pm \mathrm{SE})$ of three replicates; ${ }^{*} \mathrm{P}<0.05$.

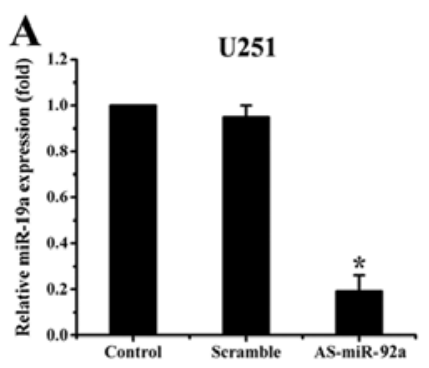

C
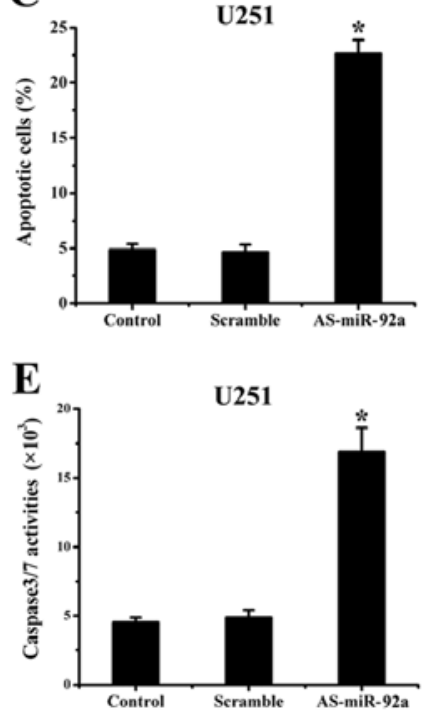
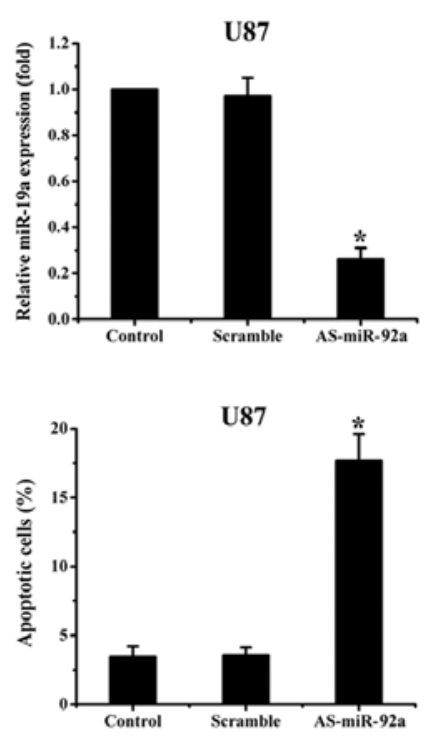

U87

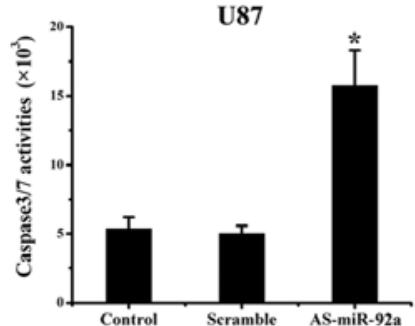

B

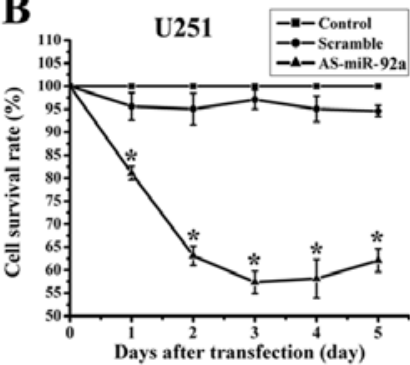

D

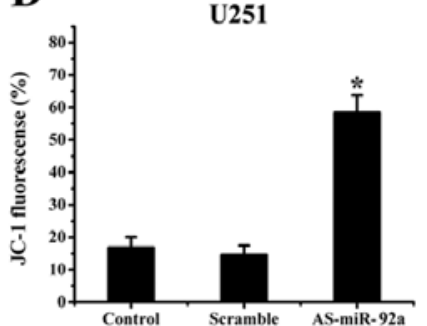

F

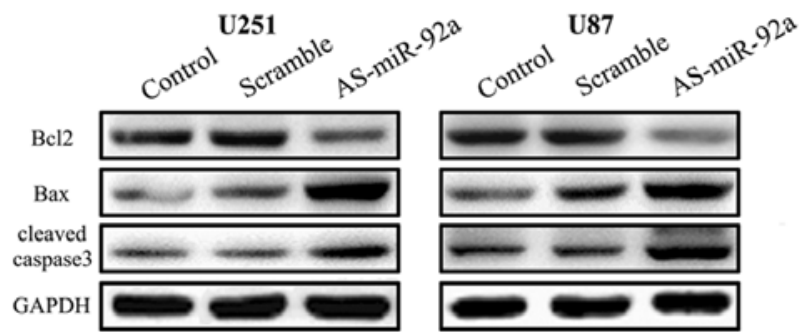

Figure 2. Downregulation of miR-92a inhibited proliferation and induced apoptosis in glioma cells. (A) miR-92a expression levels (normalized to U6 RNA) were significantly depressed by $81 \%(\mathrm{P}<0.05)$ in U251 cells and $74 \%(\mathrm{P}<0.05)$ in U87 cells treated with As-miR-92a, relative to the control. (B) MTT assay showed that AS-miR-92a suppressed the proliferative ability in U251 and U87 cells, compared with scramble and control. (C) Annexin V analysis showed that U251 and U87 cells transfected with As-miR-92a displayed significantly more apoptosis than control and scramble groups. (D) U251 and U87 cells transfected with As-miR-92a showed a significantly greater collapse in mitochondrial membrane potential compared with the other two groups. (E) A significant increase in caspase-3/7 activity was detected in U251 and U87 cells transfected with As-miR-92a. (F) U251 and U87 cells were transfected with As-miR-92a, and Bcl-2, Bax and cleaved caspase-3 protein level was detected by western blot assay. GAPDH protein was regarded as endogenous normalizer. Data are from one of three representative experiments. Data are mean $\pm \mathrm{SE}, \mathrm{n}=3 ;{ }^{*} \mathrm{P}<0.05$.

was also considerably elevated in miR-92a deleted cells (Fig. 2E). Since collapse of the mitochondrial membrane potential is one of the early events in apoptosis (22), we next examined if miR-92a regulates mitochondrial membrane potential. The cells with suppression of miR-92a were stained with cationic dye JC-1. FACSCalibur analysis showed that the mitochondrial membrane potential was largely damaged when miR-92a were downregulated (Fig. 2D). These findings indicate that miR-92a plays an important role in regulation of cell apoptosis. 
A<smiles>ClC(Cl)(Cl)C(Cl)(Cl)Cl</smiles>
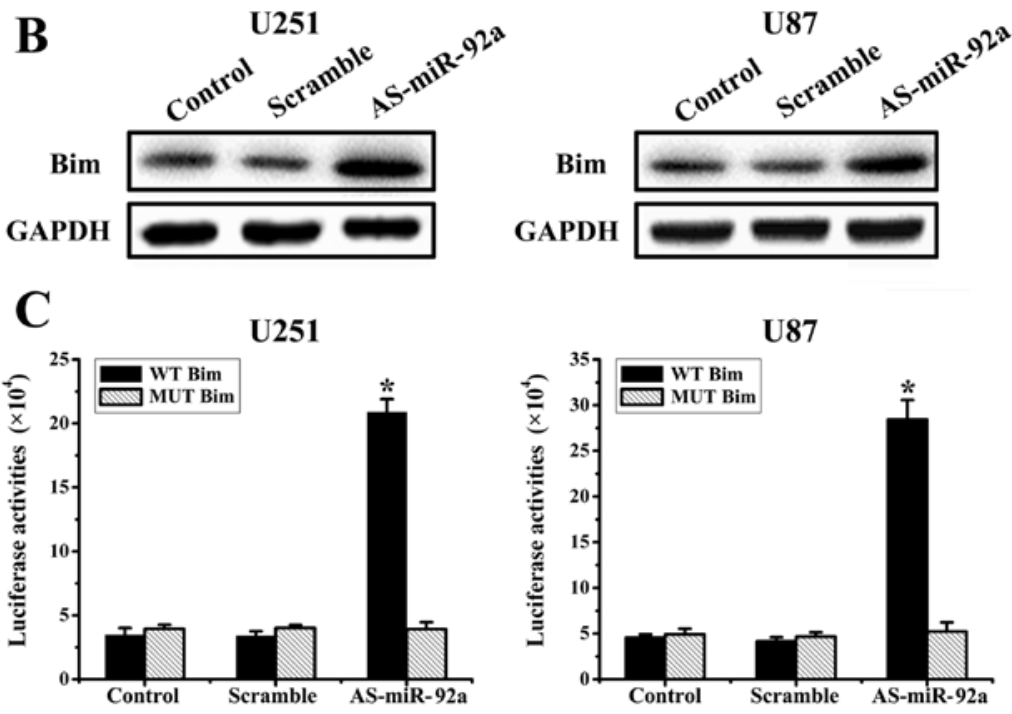

Figure 3. miR-92a directly targets Bim. (A) Bioinformation analysis identifed Bim as potential targets for miR-92a. WT Bim and MUT Bim luciferase report plasmids were constructed. (B) Western blotting for Bim at 48-h post-transfected of As-miR-92a in U251 and U87 cells. (C) Luciferase reporter assays confirmed that Bim was the direct target of miR-92a in U251 and U87 cells. Each datum represents the mean fold-change in expression from three independent experiments \pm SE.
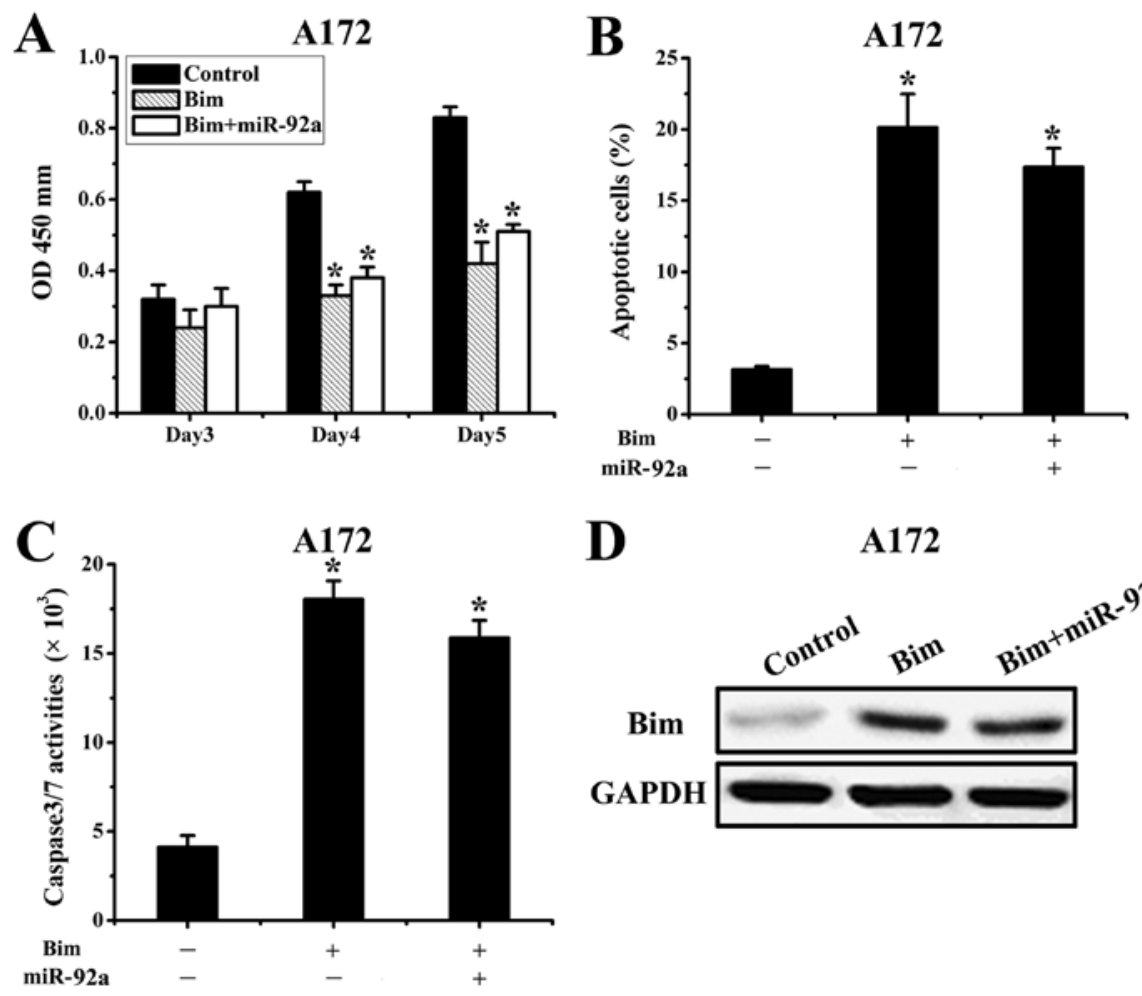

D

$\mathbf{A 1 7 2}$

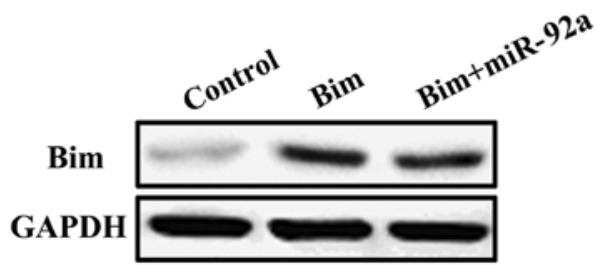

Figure 4. Expression of Bim abrogates miR-92a survival function. (A) Proliferation of A172 cells after Bim transfection with or without miR-92a were signifcantly reduced compared to control. (B) A172 cells were transfected with Bim (not including the 3'UTR) and miR-92a, and cell apoptosis was measured by Annexin V analysis. (C) Caspase activity was evaluated in A172 cells transfected with Bim (not including the 3'UTR) and miR-92a by caspase-3/7 activity assay. (D) A172 cells were transfected with Bim (not including the 3'UTR) and miR-92a, and Bim expression was measured by western blot assay. GAPDH protein was regarded as endogenous normalizer. ${ }^{\mathrm{P}} \mathrm{P}<0.05$ compared with control group. 
A
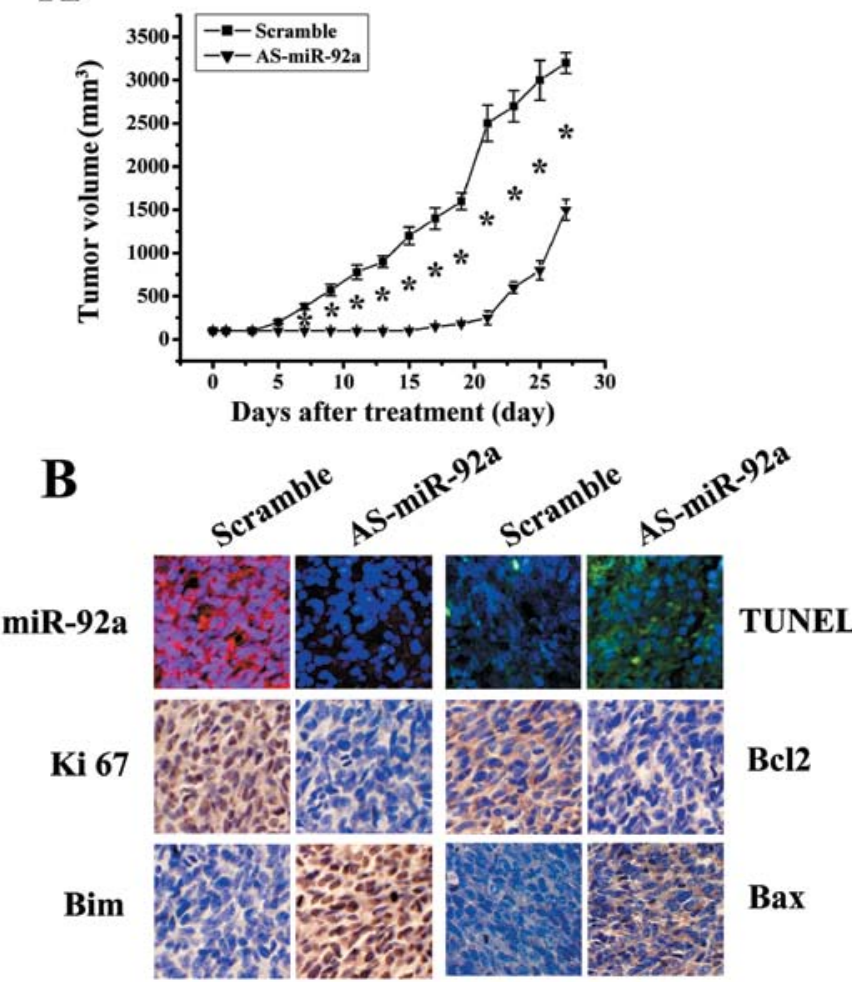

Figure 5. Knock-down of miR-92a inhibits glioma growth in vivo. (A) When subcutaneous tumors were established, As-miR-92a were injected in a multisite injection manner every 2 days for 14 days. Tumor volumes were measured every 2 days during treatment. (B) Fluorescence in situ hybridization showed that As-miR-92a effectively inhibited the expression of miR-92a; TUNEL assay in xenograft tumor sections revealed that As-miR-92a induced cell apoptosis; Ki-67, Bim, Bcl-2 and Bax expression was detected by immunohistochemistry assay in xenograft tumor sections. ${ }^{*} \mathrm{P}<0.05$ compared with scramble group.

miR-92a directly targets Bim. Based on the analysis of databases miRanda (http://www.cbio.mskcc.org/mirnaviewer), PicTar (http://www.pictar.bio.nyu.edu), and TargetScan (http://www.targetscan.org), we predicted that Bim may be a target gene for miR-92a (Fig. 3A). To determine whether Bim is regulated by miR-92a, we knocked-down miR-92a in U251 and U87 cells, and evaluated Bim protein levels at $48 \mathrm{~h}$ post-transfection. And western blot analysis showed that
AS-miR-92a obviously increased Bim protein levels in U251 and U87 glioma cells (Fig. 3B).

To assess whether Bim is a direct target of miR-92a, we created WT Bim and MUT Bim plasmids, which were co-transfected with AS-miR-92a or scrambled oligonucleotide respectively into glioma cells for $48 \mathrm{~h}$, followed by measurement of luciferase activity in transfected cells. Our results showed that the reporter plasmid with wild-type 3'UTR of Bim caused a significant increase in luciferase activity in cells transfected with AS-miR-92a, while MUT reporter plasmid produced no change in luciferase activity (Fig. 3C). Taken together, these data indicated that miR-92a directly modulates Bim expression by binding to 3'UTR of Bim.

Bim is a functional target of $m i R-92 a$. We next assessed the importance of Bim in miR-92a-mediated cell survival. Since A172 cells had relatively low expression of miR-92a, we transfected AS-miR-92a or scramble oligonucleotide $24 \mathrm{~h}$ after transfection with Bim expression plasmid lacking 3'UTR. The proliferation and apoptosis were assessed in co-transfected A172 cells, which showed that ectopic expression of Bim abrogated miR-92a effects on cell proliferation and apoptosis (Fig. 4A-C). Subsequently, western blot results demonstrated the upregulation of Bim protein levels was significantly prevented by miR-92a (Fig. 4D). Taken together, these results suggested that Bim is a critical target in miR-92a-mediated glioma apoptosis.

AS-miR-92a suppresses glioblastoma xenograft growth accompanying Bim upregulation. Since miR-92a are frequently elevated in glioblastoma and play an important role in cell survival, we further examined the effects of knockdown of miR-92a on glioma growth in vivo. As shown in Fig. 5A, AS-miR-92a significantly reduced tumor growth compared with scramble group $(\mathrm{P}<0.05)$. LNA-ISH analysis confirmed that miR-92a levels were considerably reduced in AS-miR-92a group (Fig. 5B). TUNEL assay analysis of xenograft tumor taken at 28 days after treatment revealed much more apoptosis in AS-miR-92a group when compared to tumors from scramble groups (Fig. 5B). In addition, Ki-67 staining shows that AS-miR-92a treated tumors had a lower proliferation index compared with the control groups (Fig. 5B). Immunohistochemical stanining analysis revealed that Bim

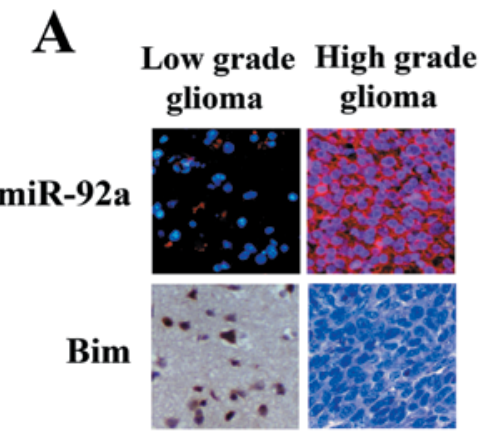

B

\begin{tabular}{ccc} 
& \multicolumn{1}{c}{$\begin{array}{c}\text { High } \\
\text { miR-92a }\end{array}$} & \multicolumn{1}{c}{$\begin{array}{c}\text { Low } \\
\text { miR-92a }\end{array}$} \\
\cline { 2 - 3 } High Bim & $8(18.2 \%)$ & $39(75 \%)$ \\
Low Bim & $36(81.8 \%)$ & $13(25 \%)$ \\
\cline { 2 - 3 } & $\mathbf{4 4}$ cases & $\mathbf{5 2}$ cases
\end{tabular}

Figure 6. Bim was downregulated in glioma samples and inversely correlated with miR-92a levels. (A) Expression of miR-92a and Bim was analyzed in representative of gliomas with LNA-ISH and immunohistochemical staining. Expression levels of miR-92a and Bim were quantified as described in Materials and methods. (B) Chi-square test analysis of miR-92a and Bim expression. The inverse correlation is significant $(\mathrm{P}<0.05)$. 
levels were upregulated in AS-miR-92a group (Fig. 5B), confirming the data in vitro that BIM as a direct target of miR-92a. Additionally, Bax expression was increased, whereas Bcl-2 expression was decreased in xenograft tumor sections (Fig. 5B). These findings further indicate that miR-92a targets Bim and that AS-miR-92a could be therapeutic means for glioblastoma intervention.

Inverse correlation of expression of miR-92a and Bim in glioma tissues. Having demonstrated Bim as a major target of miR-92a, we further investigated the correlation of between miR-92a and Bim expression in gliomas. We examined 96 human glioma specimens with LNA-ISH and immunohistochemical staining. Representative images of miR-92a and Bim are shown in Fig. 6A. Upregulation of miR-92a was detected in 44 gliomas (Fig. 6B). Of the 44 tumors with elevated miR-92a, $36(81.8 \%)$ had low levels of Bim $(\mathrm{P}<0.05)$. Thirty-nine of 52 (75\%) specimens with downregulated miR-92a presented high levels of Bim. In addition, we found that miR-92a expression increased significantly in high grade gliomas compared with low grade gliomas.

\section{Discussion}

Glioblastomas are the most malignant brain tumors of glial origin $(23,24)$. They are also among the most resistant of all human tumors to available modalities of treatment (24). Exploring the signaling pathways involved in surviving and inducing cell death of glioma cells is important for the development of more effective tumor therapies.

miRNAs have recently been described as important players in human cancer and the role of microRNA as therapeutic target has been proposed (25-28). In the present study, we focued on the upregulated miR-92a in glioma, which is a member of miR-17-92 cluster and exerts potential proliferative, anti-apoptotic, invasion-promoting effects in a variety of cancer types. Shigoka et al (14) found that miR-92a is highly expressed in hepatocellular carcinoma (HCC). In addition, the proliferation of HCC-derived cell lines was enhanced by miR-92a and inhibited by the anti-miR-92a antagomir. Chen et al (15) reported that miR-92a promotes esophageal squamous cell carcinoma (ESCC) cell migration and invasion at least partially via suppression of $\mathrm{CDH} 1$ expression, and patients with upregulated miR-92a are prone to lymph node metastasis and thus have poor prognosis. Haug et al (16) demonstrated that miRNA-92 is regulated by MYCN, and inhibits secretion of the tumor suppressor DICKKOPF-3 (DKK3) in neuroblastoma. Tsuchida et al (17) proved that miR-92a plays a pivotal role in the development of colorectal carcinoma. However, the underlying functional mechanisms in glioma remain largely unknown. In this study, we revealed that miR-92a was upregulated in specimens of human gliomas of different grades and in glioma cell lines, and high levels of miR-92a in human glioma specimens were significantly correlated with low levels of Bim protein and high histological grade. We further presented that miR-92a abgoration results in growth inhibition and apoptosis induction with upregulation of Bim in vitro and in vivo.

A mitochondrial-dependent step in apoptosis, involving mitochondrial outer membrane permeabilization (MOMP), is associated with most pro-apoptotic stimuli (29). This process is controlled by both pro- and anti-apoptotic members of the Bcl-2 family, including Bim, Bcl-2 and Bax. Reportedly, Bim can trigger apoptosis by at least two different mechanisms, by the interaction and neutralization of Bcl-2-like molecules and/or direct activation of Bax, and that the decision on which mode of action ensues perhaps depends on the nature of the incoming apoptosis signal. Low expression of Bim has been shown for tumor entities, including melanoma and renal cell carcinoma (30). The mechanism suppressing Bim in these cancer types has not been clarifed yet. In our study, we found that knockdown miR-92a could downregulate Bcl-2 and upregulate Bim and Bax by western blot assay. Furthermore, bioinformatics analysis showed that 3'UTR of Bim mRNA existed the highly conserved putative miR-92a binding sites. Luciferase reporter assay validated that Bim was a direct target of miR-92a. In addition, the change of Bim, Bcl-2 and Bax expression in xenograft study confirmed the data in vitro. These results indicate that AS-miR-92a negatively regulate Bim which leads to decrease Bcl-2 and increase Bax.

In conclusion, our experiments have shown a novel oncogenic role for miR-92a through regulation of apoptosis signaling pathways involving Bim. The resulting phenotype of an upregulated miR-92a includes increased proliferation and decresed apoptosis. Furthermore, these data raise the possibility that miR-92a may serve as a potential therapeutic target for gliomas.

\section{References}

1. Surawicz TS, McCarthy BJ, Kupelian V, et al: Descriptive epidemiology of primary brain and CNS tumors: results from the Central Brain Tumor Registry of the United States, 1990-1994. Neuro Oncol 1: 14-25, 1999.

2. Wen PY and Kesari S: Malignant gliomas in adults. N Engl J Med 359: 492-507, 2008.

3. Hadjipanayis CG and Van Meir EG: Tumor initiating cells in malignant gliomas: biology and implications for therapy. J Mol Med 87: 363-374, 2009.

4. Purow B and Schiff D: Advances in the genetics of glioblastoma: are we reaching critical mass? Nat Rev Neurol 5: 419-426, 2009.

5. Elmén J, Lindow M, Schütz S, et al: LNA-mediated microRNA silencing in non-human primates. Nature 452: 896-899, 2008.

6. Meister $\mathrm{G}$ and Tuschl T: Mechanisms of gene silencing by double stranded RNA. Nature 431: 343-349, 2004.

7. Tijsterman M and Plasterk RH: Dicers at RISC; the mechanism of RNAi. Cell 117: 1-3, 2004.

8. Kloosterman WP and Plasterk RH: The diverse functions of microRNAs in animal development and disease. Dev Cell 11: 441-450, 2006.

9. Jovanovic $\mathrm{M}$ and Hengartner MO: miRNAs and apoptosis: RNAs to die for. Oncogene 25: 6176-6187, 2006.

10. He H, Jazdzewski K, Li W, et al: The role of microRNA genes in papillary thyroid carcinoma. Proc Natl Acad Sci USA 102: 19075-19080, 2005.

11. Iorio MV, Ferracin M, Liu CG, et al: MicroRNA gene expression deregulation in human breast cancer. Cancer Res 65: 7065-7070, 2005.

12. Metzler M, Wilda M, Busch K, et al: High expression of precursor microRNA-155/BIC RNA in children with Burkitt lymphoma. Genes Chromosomes Cancer 39: 167-169, 2004.

13. Murakami Y, Yasuda T, Saigo K, et al: Comprehensive analysis of microRNA expression patterns in hepatocellular carcinoma and non-tumorous tissues. Oncogene 25: 2537-2545, 2006.

14. Shigoka M, Tsuchida A, Matsudo T, et al: Deregulation of miR-92a expression is implicated in hepatocellular carcinoma development. Pathol Int 60: 351-357, 2010.

15. Chen ZL, Zhao XH, Wang JW, et al: microRNA-92a promotes lymph node metastasis of human esophageal squamous cell carcinoma via E-cadherin. J Biol Chem 286: 10725-10734, 2011. 
16. Haug BH, Henriksen JR, Buechner J, et al: MYCN-regulated miRNA-92 inhibits secretion of the tumor suppressor DICKKOPF-3 (DKK3) in neuroblastoma. Carcinogenesis 32: 1005-1012, 2011.

17. Tsuchida A, Ohno $\mathrm{S}, \mathrm{Wu} \mathrm{W}$, et al: $\mathrm{miR}-92$ is a key oncogenic component of the miR-17-92 cluster in colon cancer. Cancer Sci 102: 2264-2271, 2011.

18. Spannuth WA, Sood AK and Coleman RL: Angiogenesis as a strategic target for ovarian cancer therapy. Nat Clin Pract Oncol 5: 194-204, 2008.

19. Arnoult D: Apoptosis-associated mitochondrial outer membrane permeabilization assays. Methods 44: 229-234, 2008.

20. Zhou X, Ren Y, Moore L, et al: Downregulation of miR-21 inhibits EGFR pathway and suppresses the growth of human glioblastoma cells independent of PTEN status. Lab Invest 90: $144-155,2010$

21. Yamamichi N, Shimomura R, Inada K, et al: Locked nucleic acid in situ hybridization analysis of miR-21 expression during colorectal cancer development. Clin Cancer Res 15: 4009-4016, 2009.

22. Yee KS, Wilkinson S, James J, et al: PUMA- and Bax-induced autophagy contributes to apoptosis. Cell Death Differ 16: $1135-1145,2009$.
23. Talotta F, Cimmino A, Matarazzo MR, et al: An autoregulatory loop mediated by miR-21 and PDCD4 controls the AP-1 activity in RAS transformation. Oncogene 28: 73-84, 2009.

24. Thaker NG and Pollack IF: Molecularly targeted therapies for malignant glioma: rationale for combinatorial strategies. Expert Rev Neurother 9: 1815-1836, 2009.

25. Nana-Sinkam SP and Croce CM: MicroRNAs as therapeutic targets in cancer. Transl Res 157: 216-225, 2011.

26. Law PT and Wong N: Emerging roles of microRNA in the intracellular signaling networks of hepatocellular carcinoma. J Gastroenterol Hepatol 26: 437-449, 2011.

27. Gandellini P, Profumo V, Folini M, et al: MicroRNAs as new therapeutic targets and tools in cancer. Expert Opin Ther Targets 15: 265-279, 2011.

28. Farazi TA, Spitzer JI, Morozov P, et al: miRNAs in human cancer. J Pathol 223: 102-115, 2011.

29. Zhang CZ, Zhang JX, Zhang AL, et al: MiR-221 and miR-222 target PUMA to induce cell survival in glioblastoma. Mol Cancer 9: 229, 2010.

30. Piñon JD, Labi V, Egle A, et al: Bim and Bmf in tissue homeostasis and malignant disease. Oncogene 27: S41-S52, 2008. 